\title{
A Scanning Acoustic Microscope Based on Picosecond Ultrasonics
}

\author{
S. Che, ${ }^{1}$ P.R. Guduru, ${ }^{2}$ A.V. Nurmikko ${ }^{1,2}$ and H.J. Maris ${ }^{1,2}$ \\ ${ }^{1}$ Department of Physics, Brown University, Providence, Rhode Island 02912 \\ ${ }^{2}$ School of Engineering, Brown University, Providence, Rhode Island 02912
}

\begin{abstract}
We report on the development of a new type of scanning acoustic microscope. We use a femtosecond light pulse to generate a short sound pulse, and then focus this sound onto the sample by means of a specially designed and microfabricated acoustic lens of radius a few microns. The sound travels to the sample through a thin layer of water. The sound reflected from the sample is collected by the lens and then passes through a monolithically integrated optical resonant cavity. The induced change in the properties of this cavity are measured using a time-delayed probe light pulse. We describe some of the challenges involved in the construction and operation of this high-precision metrology apparatus and present some preliminary results.
\end{abstract}




\section{INTRODUCTION}

The idea of acoustic microscopy dates back to 1937 when Sokolov applied for a patent for a device to detect the flaws within materials by acoustic waves. The US patent was issued in $1939^{1}$ but the application was restricted to the description of a possible design for such a microscope. Since then much progress has been made towards a useful acoustic imaging microscope. In 1959 Dunn and Fry ${ }^{2}$ reported on experiments with a simple microscope operating in transmission mode. A plane sound wave of frequency 12 $\mathrm{MHz}$ was incident on the sample and spatial variations in the transmitted intensity were measured using a thermocouple probe as a detector. Significant further progress was made by Lemons and Quate ${ }^{3}$ who constructed a scanning transmission microscope using focused ultrasound (rather than planar) at $160 \mathrm{MHz}$, together with an acoustic lens to collect the sound. Further work by this group extended the frequency range up to 4.4 $\mathrm{GHz}$ and achieved a resolution of $200 \mathrm{~nm}^{4}$

Schematic generic diagrams of transmission and reflection acoustic microscopes are shown in Fig. 1. Currently, the reflection microscope is of most interest. This geometry gives information about surface topography from the reflection of the sound at the interface between the liquid coupling medium (CM) and the sample. The sound will also penetrate into the sample and consequently elastic properties of the material near to the sample surface can be studied.

The resolution $d$ of an acoustic microscope is related to the sound wavelength $\lambda$ by the formula

$$
d=\frac{0.51 \lambda}{N A},
$$

where $N A$ is the numerical aperture. However, the attenuation of sound in most liquids, (water is the CM normally used), increases as the square of the frequency. As a result, for a given path length between the lens and the sample, there is a maximum frequency which can be used, and a minimum wavelength which limits the spatial resolution. To reduce the wavelength and to improve the resolution it is therefore necessary to decrease 
the travel distance through the CM. In water, the attenuation coefficient $\alpha$ per unit distance is $\beta f^{2}$ where $f$ is the frequency and $\beta=19 \times 10^{-17} \mathrm{sec}^{2} \mathrm{~cm}^{-1}$ at $30 \mathrm{C}{ }^{5}$ Therefore, the amplitude of a $10 \mathrm{GHz}$ sound wave is reduced by a factor of $\exp (-1.9) \simeq 0.15$ after traveling $1 \mu$. Because the attenuation is proportional to frequency squared, the attenuation of the high frequency components results in a pulse profile which is a Gaussian with full width at half maximum of $\frac{2 \sqrt{\ln 2}}{\pi} v \sqrt{\beta L}$ where $v$ is the sound velocity and $L$ is the distance traveled. When $L=1 \mu$, this width is $0.11 \mu$, and for $0.3 \mu$ it is $60 \mathrm{~nm}$.

Decreasing the travel distance requires the use of a very short sound pulse. This is because there is always substantial reflection of sound at the interface between the focusing/collecting acoustic lens and the CM. Thus, in the reflection acoustic microscope, for example, the signal of interest comes from the sound which has been transmitted into the $\mathrm{CM}$, reflected at the surface of the sample, and then passed across the interface back into the lens. There will also be an unwanted signal of comparable magnitude which has been reflected at the CM to lens interface and then bounced off the sample a second time. To separate these in time, the total length of the sound pulse when it is in the CM has to be significantly less than the diameter of the lens. With conventional ultrasonic techniques using electrically-driven piezoelectric transducers, it is very difficult to generate sound pulses shorter than a few nanoseconds, and this limits the smallest possible travel distance. However, it is possible to use light pulses to generate and detect much shorter sound pulses, ${ }^{6}$ and for this reason we have used this technique in conjunction with specially designed and monolithically integrated optoacoustic focusing transceivers. To fully employ this technique for a practical acoustic microscope requires the solution of a number of experimental problems and this is the focus of the present paper.

\section{EXPERIMENT}


In the picosecond ultrasonic technique a short light pulse is absorbed in a metal or semiconductor sample thereby setting up a thermal stress near the surface. The stress relaxes and a strain pulse is generated which propagates into the sample. When the strain pulse reaches an interface it is partially reflected, and the reflected component returns to the surface. This results in a change in the optical reflectivity of the surface which is measured with a time-delayed probe light pulse. The technique makes possible ultrasonic experiments on thin films, and attenuation measurements have been made at frequencies up to several hundred $\mathrm{GHz}{ }^{7}$

\section{a) Scanning Microscope design}

The design of the microscope is shown schematically in Fig. 2. The structure for generating and detecting sound is fabricated on the lower surface of a $500 \mu \mathrm{m}$ glass wafer. An optical cavity is formed by a $230 \mathrm{~nm} \mathrm{SiO}{ }_{2}$ film which is sandwiched between a $7 \mathrm{~nm} \mathrm{Al} \mathrm{film} \mathrm{deposited} \mathrm{on} \mathrm{the} \mathrm{substrate} \mathrm{and} \mathrm{a} \mathrm{lower} 50 \mathrm{~nm} \mathrm{Al} \mathrm{film.} \mathrm{The} \mathrm{acoustic} \mathrm{lens} \mathrm{is}$ fabricated within a $1.8 \mu \mathrm{m} \mathrm{Al}$ or $\mathrm{SiO}_{2}$ film deposited below the optical cavity by a method we describe below.

The optical cavity is necessary to improve the signal-to-noise ratio in the experiment. The changes in optical reflectivity in the all-optical picosecond ultrasonics experiments to study sound propagation in solids are small, typically of the order of $10^{-5}$, but can be measured without much difficulty. However, in an acoustic microscope the signal is reduced because of the partial transmission of sound across interfaces and because of the attenuation. A sound pulse going from medium 1 to medium 2 and then returning to medium 1 is reduced in amplitude by the factor $4 Z_{1} Z_{2} /\left(Z_{1}+Z_{2}\right)^{2}$, where $Z_{1}$ and $Z_{2}$ are the acoustic impedances. For aluminum and water $Z_{1} / Z_{2}=11.7$ and so the amplitude is reduced by a factor 0.29 . The use of an optical cavity has been described in earlier papers by our group ${ }^{8,9}$. The optical cavity increases the absorption of the generating pump light pulse (relative to the absorption which would occur for a single Al film), thereby increasing the amplitude of the generated sound. When the sound pulse 
returns after propagating through the water and being reflected at the sample, it results in a change in the cavity spacing and an enhanced change in reflectivity of the probe light. As discussed previously, ${ }^{8,9}$ the optimization of the design for the cavity has to take into account several factors:

1) As far as the choice of materials is concerned, it is advantageous to choose all materials in the cavity structure with nearly the same acoustic impedance. This is to minimize reflection of sound at the interfaces. The amplitude reflection coefficient at a $\mathrm{SiO}_{2} / \mathrm{Al}$ interface is $\sim 10 \%$.

2) In order to maximize the absorption of the pump it would be best to make the width of the cavity such as to give a minimum reflectivity. However, if the width were chosen this way there would be no change in the reflectivity of the probe light (assuming this had the same wavelength as the pump) when the sound changed the cavity spacing.

3) A high $Q$ factor of the cavity increases the change in reflectivity due to a change in spacing. However, in order to focus the pump and probe beams onto the area above the acoustic lens it is necessary to use a high numerical aperture lens. Thus, these beams are composed of light with a range of propagation directions and each direction needs a slightly different cavity spacing to be in resonance. Thus, there is a limit to the highest $\mathrm{Q}$ that can be used.

In the current experiment we have used a 60x objective with focal length $1 \mathrm{~cm}$ to focus both the pump and probe beams. The diameters of the beams incident on the lens were approximately $3 \mathrm{~mm}$. This setup made it possible to focus onto a spot on the acoustooptic cavity of about $3 \mu \mathrm{m}$ diameter. The laser source had wavelength $800 \mathrm{~nm}$, with pulse width of $200 \mathrm{fs}$ and $80 \mathrm{MHz}$ repetition rate. The spectral width of the laser output is approximately $12 \mathrm{~nm}$. We used two laser line filters to set the pump and probe beams at slightly different wavelengths; $809 \mathrm{~nm}$ for the pump and $806 \mathrm{~nm}$ for the probe. This made it possible to have the probe at a wavelength where the reflectivity of the cavity changed most rapidly with spacing, while the pump had a wavelength at which the absorption of energy was higher. The pump and probe beams had powers of $7 \mathrm{~mW}$ and 4 $\mathrm{mW}$, respectively, and the pulse energy applied per unit area was about 1 and $0.5 \mathrm{~mJ}$ $\mathrm{cm}^{-2}$, respectively. 
The remainder of the experimental setup follows the standard pump-probe measurement technique for ultrasonics. ${ }^{6}$ The pump beam was modulated by an electro-optic modulator at $1 \mathrm{MHz}$, and the output of the detector of the reflected probe light was fed to a lock-in amplifier using the $1 \mathrm{MHz}$ as reference. A third laser line filter passing $806 \mathrm{~nm}$ was put in front of the probe photo-detector to reduce the effects of scattered pump light.

To scan the lens across the sample we used the translation stage shown in Fig. 3. In the figure, part $\mathrm{A}$ is a square " $\mathrm{A}$ " shaped frame bolted onto the bottom plate $\mathrm{E}$. On the top center of $\mathrm{A}$, there is a holder for the structure comprised of the optical cavity and acoustic lens; this has an opening for access of the pump and probe laser beams coming from above. The sample to be tested is mounted on the sample holder B with its surface aligned approximately parallel to the bottom of the optical cavity/acoustic lens structure. The sample holder is bolted onto a P-517.3CL nano-positioning stage $\mathrm{C}$ from Physik Instrumente. ${ }^{10}$ This has a $100 \mu \mathrm{m}$ moving range in the $x$ and $y$ directions and $20 \mu \mathrm{m}$ in the $z$ direction. In addition, a coarse adjustment of the sample height with moving range of about $1 \mathrm{~mm}$ and 0.02 radians in the tip-tilt angle was provided by a Kelvin clamp between the plate $\mathrm{D}$ and the base plate $\mathrm{E}$.

\section{b) Design and Fabrication of the Integrated Acoustic Lens}

For this experiment we needed to have a concave acoustic lens and wanted to make the numerical aperture as large as possible. Consider first the design of an aluminum lens. The construction of the lens is made easier because of the large difference between the sound velocity $v_{1}$ in aluminum $\left(6.42 \times 10^{5} \mathrm{~cm} \mathrm{~s}^{-1}\right)$ and the velocity $v_{2}$ in water ( $1.49 \times 10^{5} \mathrm{~cm} \mathrm{~s}^{-1}$ at $23 \mathrm{C}$ ). This difference reduces the effects of spherical aberration. Let the axis of the lens coincide with the $z$-axis, and the location of the lens surface be $z=\zeta(r)$ where $r$ is the distance from the $z$-axis, with $\zeta(0)=0$. In the experiment an essentially planar sound pulse propagates in the positive $z$-direction. Based on geometrical optics (actually geometrical acoustics), one can show that in order for all of 
the incident sound to come to a focus at the on-axis location $r=0, z=f$, the lens has to be aspherical with profile

$$
\zeta(r)=\frac{v_{1}}{v_{1}+v_{2}}\left[f-\sqrt{f^{2}-\frac{v_{1}+v_{2}}{v_{1}-v_{2}} r^{2}}\right] .
$$

For small values of $r$

$$
\zeta(r)=\frac{v_{1}}{2\left(v_{1}-v_{2}\right)} \frac{r^{2}}{f} .
$$

Thus the radius of curvature of the region of the lens near to the axis is

$$
R=\frac{v_{1}-v_{2}}{v_{1}} f
$$

As an example, we show in Fig. 4 the calculated profile for an aspheric lens with $f=2$ $\mu \mathrm{m}$, along with the profile of a spherical lens of radius given by Eq. 4, i.e., $R=1.537$ $\mu \mathrm{m}$. One can see that the difference between these two lens profiles is very small. In addition, the transmission of sound from aluminum to water decreases rapidly as the angle of incidence to the lens surface increases making the contribution to the signal at the focus from the outer part of the lens unimportant. For these reasons we have not tried to make lenses with non-spherical profiles.

A wide range of techniques has been used for the fabrication of microlenses for optical applications. ${ }^{11}$ Convex lenses can readily be made by deposition of a thin film, patterning into dots, and followed by reflow. ${ }^{12}$ Concave lenses have been made by first depositing a thin metal film onto a substrate, making a small hole in the film, and then wet etching the substrate through the hole. ${ }^{13}$ We attempted to make lenses directly on a $\mathrm{SiO}_{2}$ substrate using this method but the lenses did not have an acceptable (ideal) profile.

We therefore developed a process for lens fabrication based on nanoindentation of aluminum. The simplest approach would be to press the nanoindenter tip directly into a metal film. However this would require an indenter tip with a shape which was accurately spherical. Instead of using a tip like this, we used a tip with an almost flat end face to press a silica sphere into an aluminum film. We first deposited an approximately $2 \mu \mathrm{m}$ aluminum film using electron beam evaporation. This film was then patterned into 
round islands with diameters of $10,15,20,25$ or $30 \mu \mathrm{m}$ by photolithography. Having different size islands made it easier to locate a particular island after nanoindentation. A drop of water containing about $10^{7}$ silica spheres per $\mathrm{cm}^{3}$ was then put on the film, and the water was then allowed to slowly evaporate. This left a single layer of spheres attached to the islands by the van der Waals force, as shown in Fig. 5a. After indentation using a Hysitron nanoindentor with diamond tip TI-046, ${ }^{14}$ we removed the spheres by sonicating the sample in water. We have made lenses with radius of curvature between 0.5 and $2.5 \mu \mathrm{m}$ in this way, and can control the numerical aperture through the choice of the depth that the sphere goes into the film. This method has the advantage that the indentation reduces the roughness of the surface of the film. An example of lenses made in this way is shown in Fig. 5b. The result of an AFM measurement of the profile of a lens made using a $2.5 \mu \mathrm{m}$ sphere is shown in Fig. 6 .

The nanoindentation method can only be used with ductile materials. To make lenses in silicon dioxide we have used a focused ion beam (FIB). ${ }^{15} \mathrm{We}$ first prepared the $\mathrm{Al}$ and $\mathrm{SiO}_{2}$ films for the optical cavity. We then sputtered onto the outer $\mathrm{Al}$ film a $700 \mathrm{~nm}$ layer of $\mathrm{SiO}_{2}$. A thin layer ( $5 \mathrm{~nm}$ thickness) of $\mathrm{Cr}$ was next deposited by e-beam evaporation onto this $\mathrm{SiO}_{2}$ film. We then deposited some small platinum target patterns onto the $\mathrm{Cr}$ using Ga-ion beam assisted chemical vapor deposition of a precursor organometallic gas. These patterns served as markers which were used to optimize the focusing of the $\mathrm{Ga}$ ion beam without modifying the remainder of the surface. We then proceeded to sculpt the lens using a Ga beam voltage of $30 \mathrm{kV}$ and current $48 \mathrm{pA}$. An electron beam was also used for imaging and monitoring the fabrication process. After finishing milling, we measured the actual profile of the lens using an atomic force microscope. The FIB milling rate was found to be about $0.24 \mu \mathrm{m}^{3}$ per nano Coulomb. We then modified the milling time to obtain the desired lens profile. A scanning electron microscope image of a lens of radius $1.5 \mu \mathrm{m}$ made by FIB is shown in Fig. 7. In principle, this method should give greater control of the lens profile but we have not yet investigated the limits of this in detail.

\section{c) Performance Simulations of the Acoustic Microlenses}


To understand better the focusing action of the microlens we wrote a finite-difference time-domain FORTRAN program to simulate the sound propagation in the system. In order to concentrate on just the action of the lens, we did not consider the acoustics of the adjacent optical cavity, i.e., we took all of the material above the lens to be aluminum. In the initial condition the particle displacement and velocity were zero everywhere and there was a thermal stress in the layer of the aluminum lying between 1490 and $1740 \mathrm{~nm}$ above the planar interface between the aluminum and the water. The program used isotropic linear elasticity for the aluminum and for sound propagation in the water included the effects of both shear and bulk viscosity. Absorbing boundary conditions were applied at all of the boundaries of the region in which the simulation was performed. To create these conditions we used the perfectly matched layer method introduced by Berenger. ${ }^{16}$ As an example, we considered a lens with radius of curvature $2.5 \mu \mathrm{m}$ and which subtended a semi-angle of 45 degrees. Results are shown in Fig. 8 for selected times; part a) shows the strain distribution before the sound has reached the top of the lens, b) is at a time just after the sound has crossed into the water, and c) and d) show the strain distribution just before and just after the sound reaches the acoustic focus. The figure shows the volume strain $\eta$ as a linear plot.

\section{d) Benchtop Experimental Proof-of-Concept Results}

In order to make measurements, the pump and probe beams have to be focused onto the area of the optical cavity directly above the lens. This is challenging because of the small size of the lens. To find the correct position we first direct the light beams to the general area of the lens; unless we are very fortunate this means that the beams are incident at a position above an unpatterned flat region of the thick aluminum film (see Fig. 2). At this first stage of the assessment we have not yet introduced the water between this film and the sample. We then scan the time-delay of the probe beam relative to the pump spot and in this way detect the echo from the flat and free surface of the aluminum film. The result of the pump-probe measurement is shown as the dashed curve in Fig. 9. In this plot the data have been processed to remove the contribution from Brillouin oscillations arising 
from sound propagating back in the glass substrate. ${ }^{17}$ The echo arrives at around $t_{1}=620$ ps. The time-delay of the probe is then set fixed at this time and the lens structure is scanned in $x$ and $y$. This scanning was typically done in $0.5 \mu \mathrm{m}$ steps and over a $15 \mu \mathrm{m}$ range. When the pump and probe beams are directly above the lens, the echo from the flat and free surface is reduced in amplitude; it does not completely disappear because the area illuminated by the pump and probe beams extends somewhat beyond the area of the lens. At the same position a new and earlier echo appears at time $t_{2}$ which comes from sound reflected from the top of the lens; this can be seen in the solid curve in Fig. 9. In Fig. 10 we show a grey scale plot of the signal strength at time $t_{2}$ as a function of position $x y$.

After locating the lens we then add the water coupling medium between the lens and the sample. The height of the sample is adjusted and the acoustic echo from the sample is detected. The sample is then translated laterally while the time delay of the probe light pulse is held constant and the height of the echo recorded. A scan is typically performed over a distance of $2 \mu \mathrm{m}$ with a step size of $10 \mathrm{~nm}$. In the experiment, signal to noise is a major problem and because of the required signal averaging even a scan over such a short distance and in one dimension takes 5 minutes.

For these first proof-of-concept experiments we have used the acoustic microscope to look at two samples. The first was a glass grating consisting of a series of trenches of width $270 \mathrm{~nm}$, depth $220 \mathrm{~nm}$, and repeat distance $550 \mathrm{~nm}$, and measurements were made using an aluminum lens. Results are shown in Fig. 11a. A Fourier analysis of the signal as a function of $x$ gives a peak at a period of $540 \mathrm{~nm}$ in reasonable agreement with the sample period as measured by scanning electron microscopy.

The second sample was also based on bulk $\mathrm{SiO}_{2}$ and had a period of $550 \mathrm{~nm}$, with grooves of depth $450 \mathrm{~nm}$. An SEM image is shown in Fig. 12. For this sample we used an $\mathrm{SiO}_{2}$ acoustic lens made by the focused ion beam method. The lens had a radius of $2.5 \mu \mathrm{m}$. The data are shown in Fig. 11b and the period giving a best fit to the data was found to be $520 \mathrm{~nm}$. 


\section{SUMMARY}

In this paper we have outlined the concept, modeling by simulations, and initial experimental results for a proof-of-concept demonstration of a new type of highfrequency all-optical scanning acoustic microscope. In so doing we have built on our group's prior experience with picosecond ultrasonics while constructing a first version of a prototype instrument. A particularly critical new innovation has been the design and development of fabrication methods of an opto-acoustic micro-scale element which combines an acoustic focusing lens with an optical Fabry-Perot cavity. The performance of the lens was first simulated by numerical methods, to guide its experimental design in terms of the critical parameters. As for the lens fabrication, we have initially identified and implemented two rather different approaches, which give a useful basis for further development and improvement, e.g., of the control of the lens profile (curvature) and satisfying the nanoscale smoothness requirements. In terms of the overall scanning acoustic microscope instrument, work reported here has been limited to bench top demonstration of scanning surface profiles of grating-type surface corrugated $\mathrm{SiO} 2$ samples, with approximately $100 \mathrm{~nm}$ spatial resolution.

In the work performed so far we have certainly not reached the fundamental limits for the performance of this type of instrument. The present setup suffers from inadequate stability of the mechanical stage and poor signal to noise ratio. It should be straightforward to improve stability of the stage. To improve the signal to noise, an improved detection system is required. The present optical cavity constructed using aluminum and silicon has a quality factor $Q$ of only 7. The $Q$ of the cavity could be greatly improved by using multiple dielectric films as the reflecting elements. If the pump and probe beams had different wavelengths, a separate absorbing film outside the cavity could be used to generate the sound.

The numerical simulations, suggest that a scanning instrument with resolution perhaps to $50 \mathrm{~nm}$ could be possible. An acoustic microscope of this type could be used for 
measurements of surface topography and also for measurements of the elastic properties of the material near to the surface of a sample, just as in a conventional acoustic microscope. Such a tool could have multiple applications for nondestructive metrology of complex structural features of semiconductor integrated circuits during large-scale waferlevel fabrication.

We thank Kwangdong Rho for help with the lens fabrication. This work was supported in part by the Department of Energy under Grant No. DE-SC0001988, Air Force Office of Scientific Research under Contract No. FA9550-08-1-0340, and by Zygo, Inc. PRG acknowledges DOE EPSCoR Implementation Grant No. DE-SC0007074 which supports the nanoindentor facility.

\section{REFERENCES}

1. S.Y. Sokolov, U.S. Patent 2,164,265 "Means For Indicating Flaws in Materials," publication date June 27, 1939.

2. F. Dunn and W.J. Fry, 31, 632 (1959).

3. R.A. Lemons and C.F. Quate, Appl. Phys. Lett, 24, 163 (1974).

4. B. Hadimioglu and C.F. Quate, Appl. Phys. Lett. 43, 1006 (1983).

5. J. Rouch, C.C. Lai, and S.H. Chen, J. Chem,. Phys. 65, 4016 (1976).

6. C. Thomsen, H.T. Grahn, H.J. Maris and J. Tauc, Phys. Rev. B34, 4129 (1986).

7. C.D. Zhu, H.J. Maris and J. Tauc, Phys. Rev. B 44, 4281 (1991).

8. F. Yang, T.J. Grimsley, S. Che, G.A. Antonelli, H.J. Maris and A.V. Nurmikko, J. Appl. Phys. 107, 103537 (2010).

9. T.J. Grimsley, F. Yang, G.A. Antonelli, H. J. Maris, A. V. Nurmikko, Chinese Journal of Physics, 49, 151 (2011).

10. Physik Instrumente, 16 Albert St, Auburn, MA 01501.

11. H. Ottevaere, R. Cox, H.P. Herzig, T. Miyashita, K. Naessens, M. Taghizadeh, R. Völkel, H.J. Woo, and H. Thienpont, J. Opt. A 8, S407 (2006). 
12. C.S. Lim, M.H. Hong, Y. Lin, , Q. Xie, B.S. Luk'yanchuk, A. Senthil Kuma, and M. Rahman, Appl. Phys. Lett. 89, 191125 (2006).

13. C.S. Lim, M.H. Hong, A. Senthil Kuma, M. Rahman and X.D. Liu, Int. J. Machine Tools and Manufacture 46, 552 (2006).

14. Triboindentor T900 from Hysitron, Inc., Minneapolis, MN 55344.

15. Helios 600 from FEI, Hillsboro, Oregon 97124.

16. J.P. Berenger, J. Comp. Phys., 114, 185 (1994).

17. The origin and theory of Brillouin oscillations is discussed in H.N. Lin, R.J. Stoner, H.J. Maris and J. Tauc, J. Appl. Phys., 69, 3816 (1991). 


\section{FIGURE CAPTIONS}

Fig. 1. Schematic diagram of (a) transmission and (b) reflection acoustic microscopes.

Fig. 2. Schematic diagram of the scanning opto-acoustic microscope.

Fig. 3. Translation stage. The functions of the different components are described in the text.

Fig. 4. Comparison between the shape of an aspheric lens and a spherical lens.

Fig. 5. (a) Optical image of $5 \mu \mathrm{m}$ silica spheres on a $20 \mu \mathrm{m}$ diameter aluminum island.

(b) Optical image of two acoustic lenses formed after indentation and removal of silica spheres on a $15 \mu \mathrm{m}$ diameter island.

Fig. 6. Profile of a lens made using a $2.5 \mu \mathrm{m}$ silica sphere. The $x$-axis is along a line passing through the center of the lens.

Fig. 7. Scanning electron microscope image of a $\mathrm{SiO}_{2}$ lens of radius $1.5 \mu \mathrm{m}$.

Fig. 8. Results of a computer simulation of the sound propagation for a $2.5 \mu \mathrm{m}$ radius lens with a $45^{\circ}$ semi-angle. The volume strain is plotted on a linear scale, and is shown for times of $50 \mathrm{ps}, 300 \mathrm{ps}, 2070 \mathrm{ps}$ and $2480 \mathrm{ps}$.

Fig. 9. Pump-probe data used to locate the acoustic lens. The dashed curve was obtained with the pump and probe beams at a position such that the sound is not incident on the acoustic lens. An echo is seen at time $t_{1}$. The solid curve was obtained with the pump and probe beams directly above the lens resulting in an echo from the top of the lens. An additional echo is now seen at time $t_{2}$.

Fig. 10. Grey scale plot of the strength of the echo from the top of the lens as a function of position of the acoustic lens.

Fig. 11. Data obtained from a lateral scan over the samples described in the text. a) Data obtained on a trench sample with an aluminum lens, and b) data obtained with an $\mathrm{SiO} 2$ lens from the sample with profile shown in Fig. 12.

Fig. 12. SEM profile of the second $\mathrm{SiO}_{2}$ sample. Data obtained on this sample are shown in Fig. 11b. 


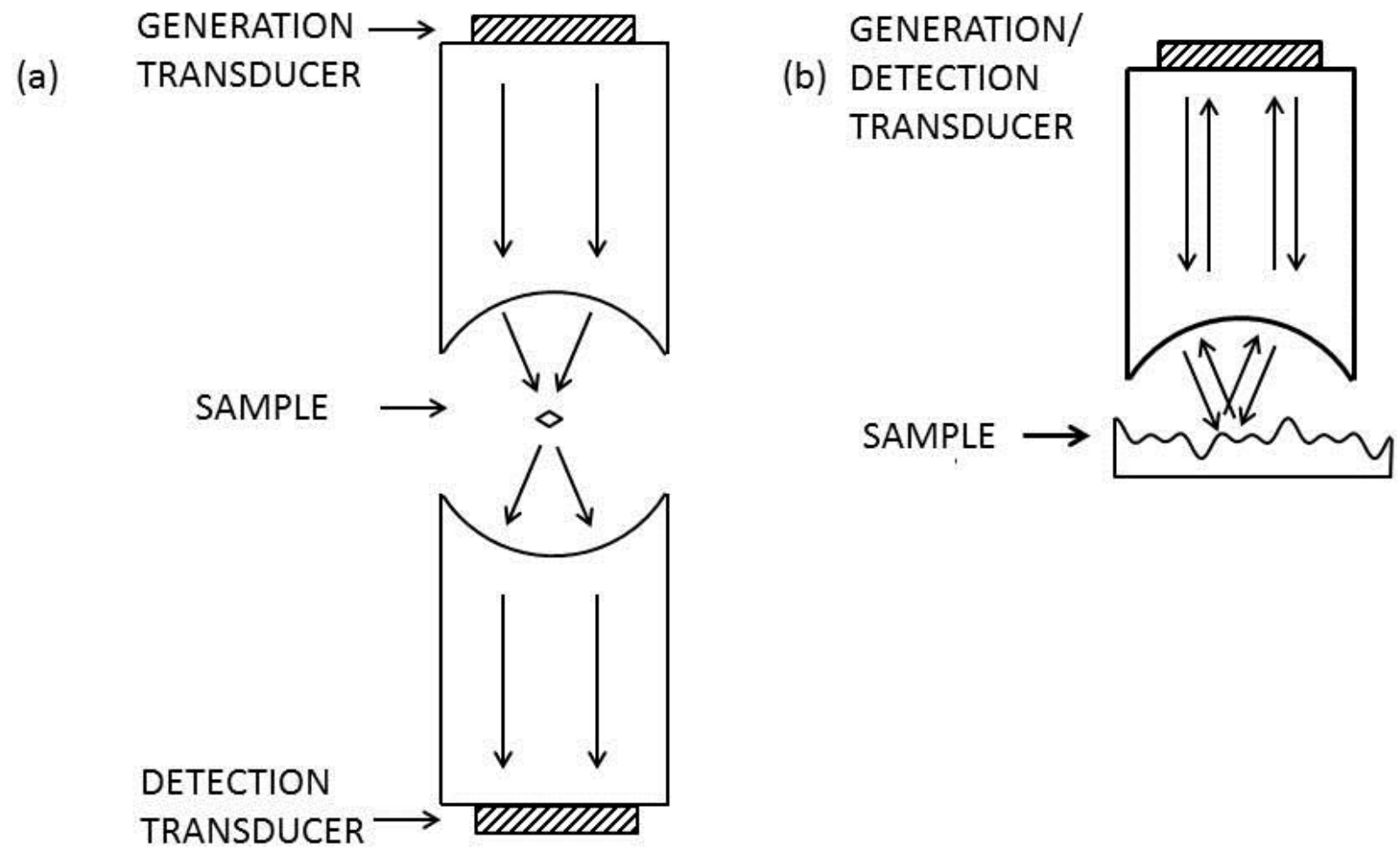

Fig. 1

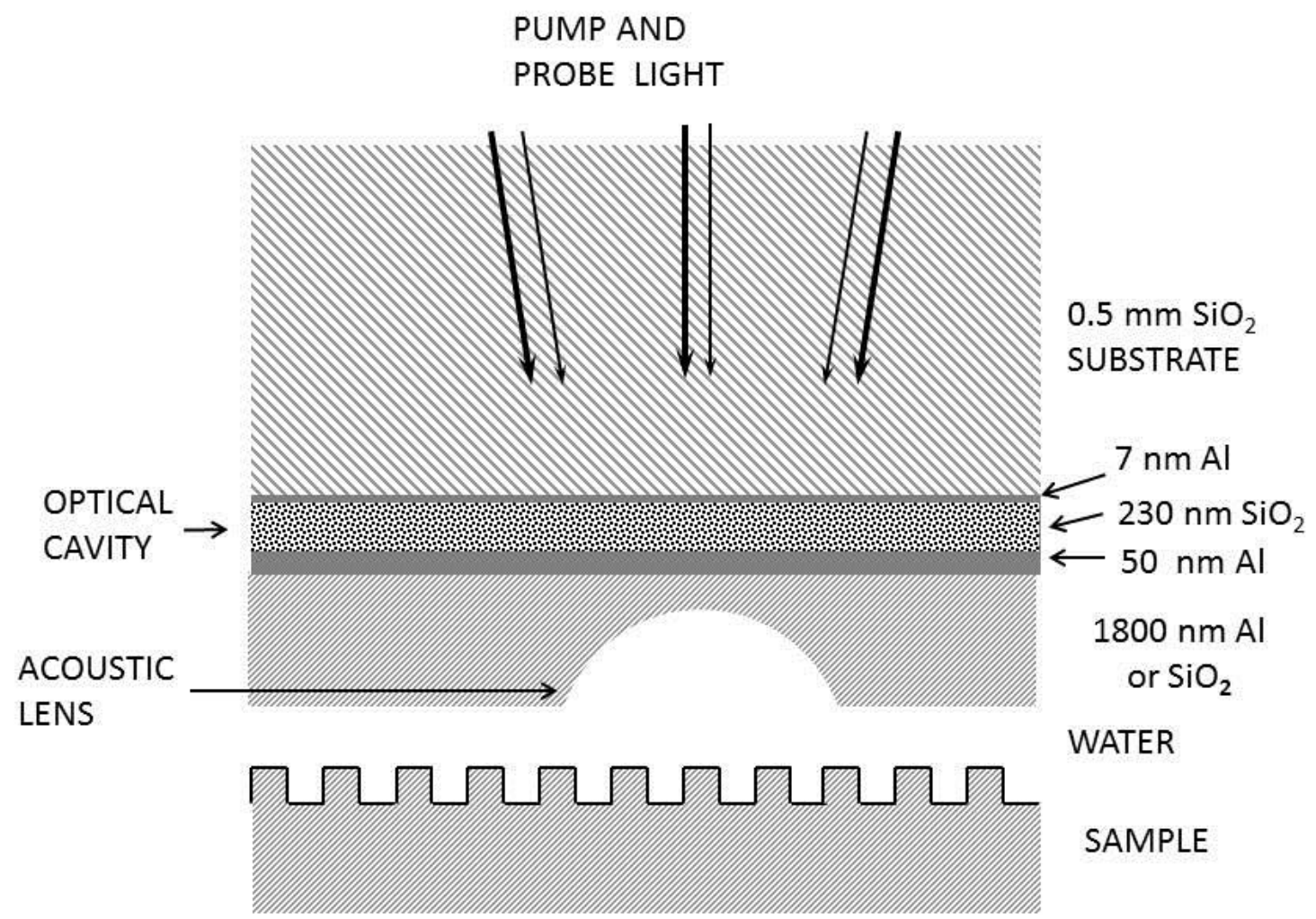

Fig. 2 


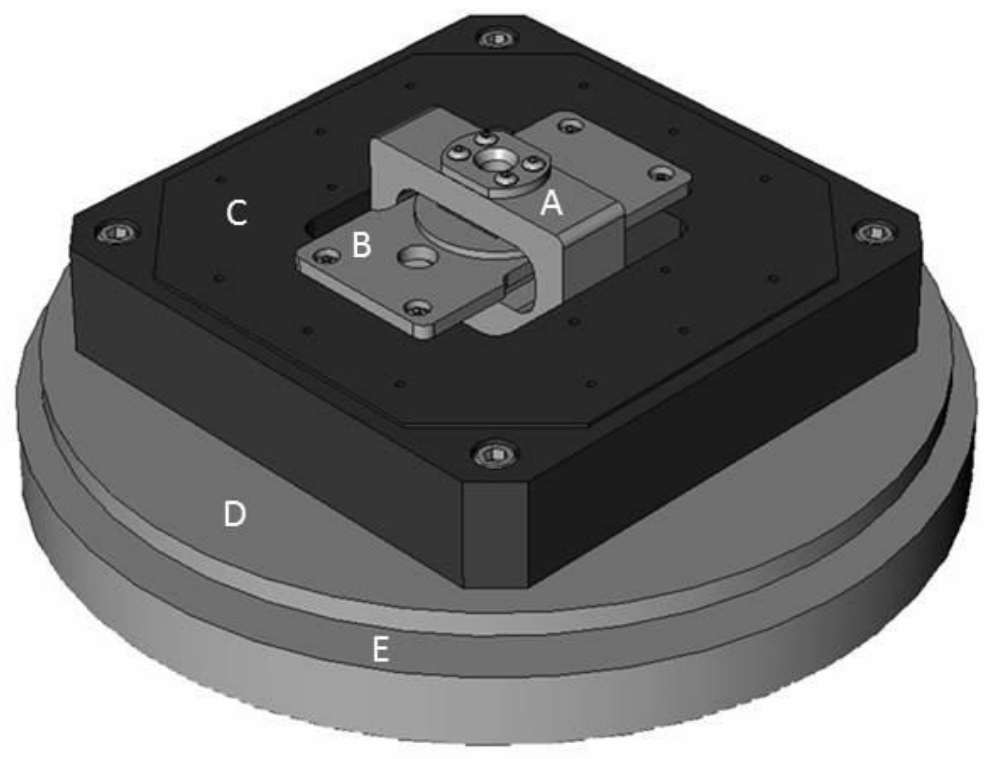

Fig. 3

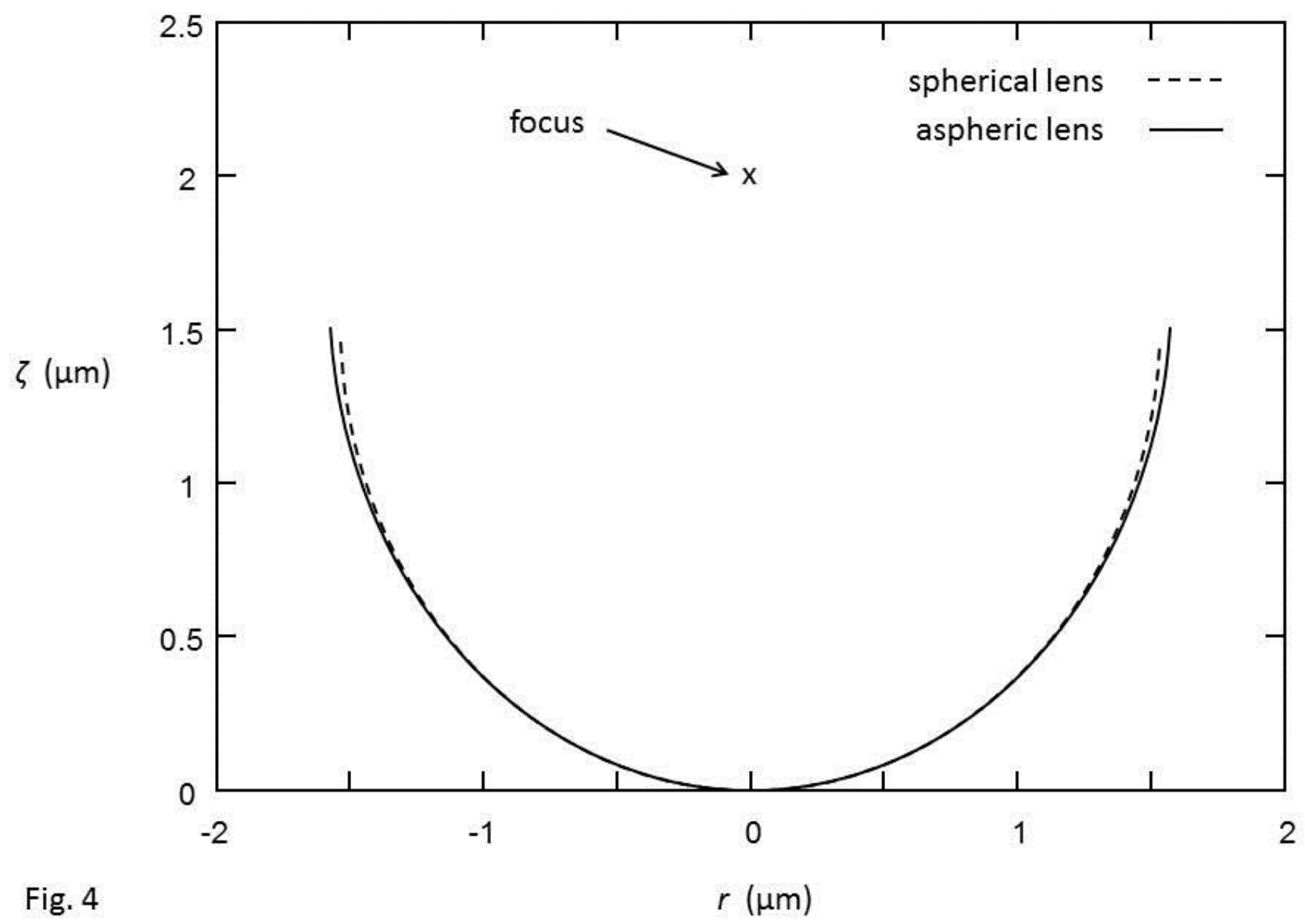



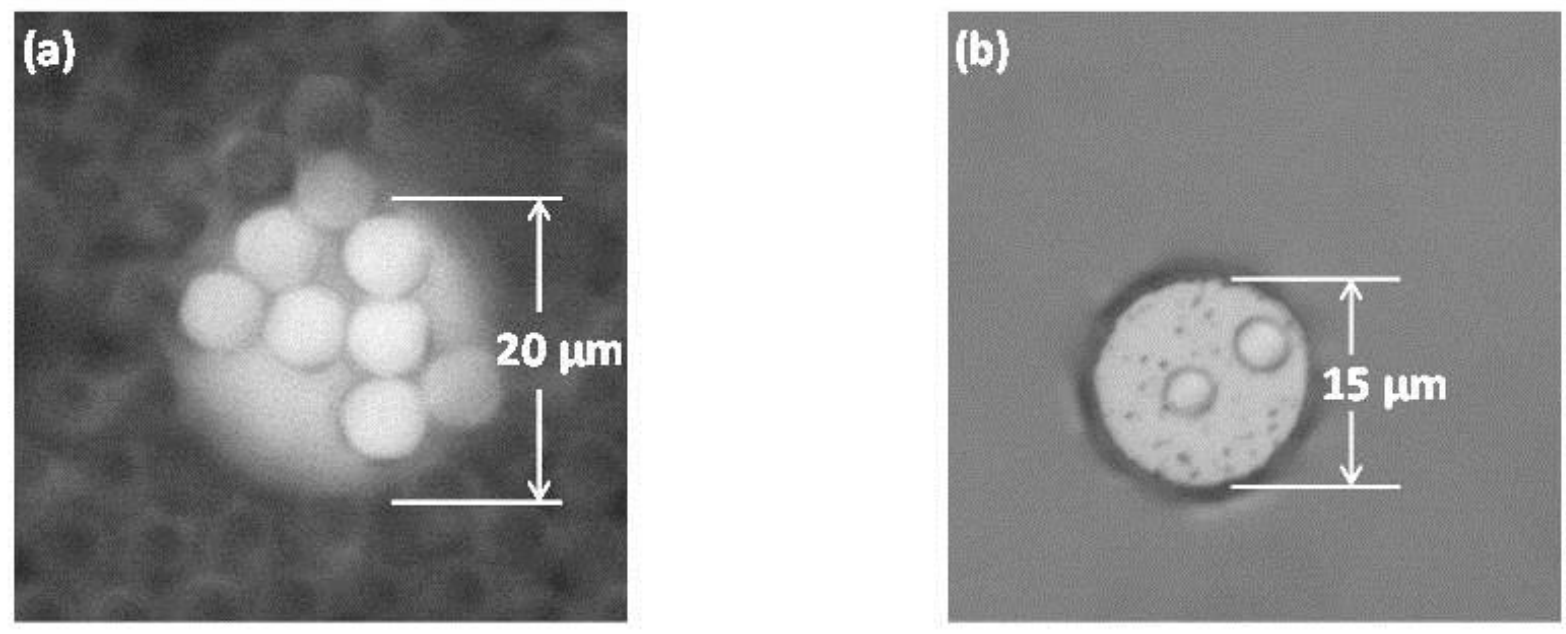

Fig . 5

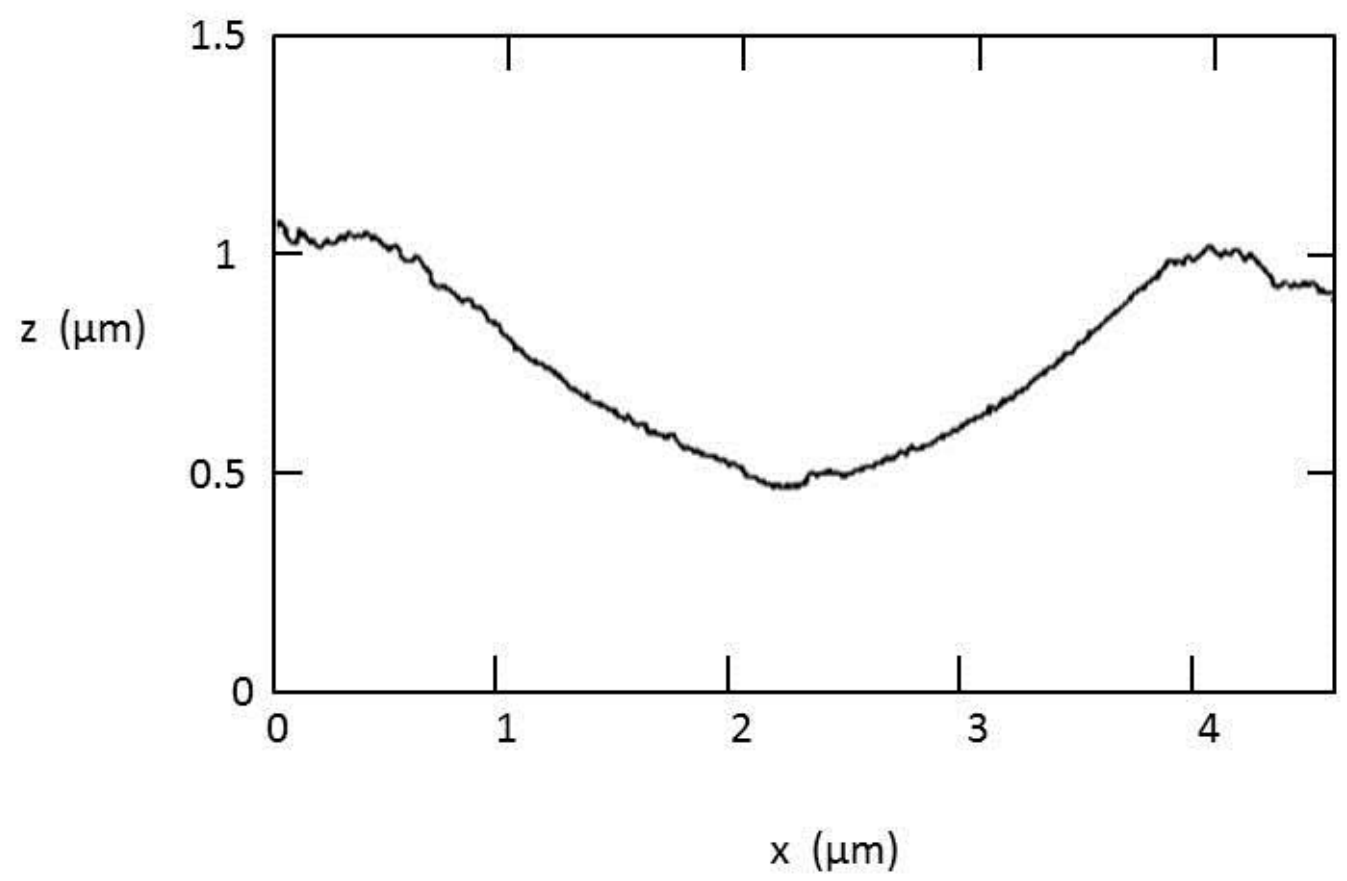

Fig. 6. 


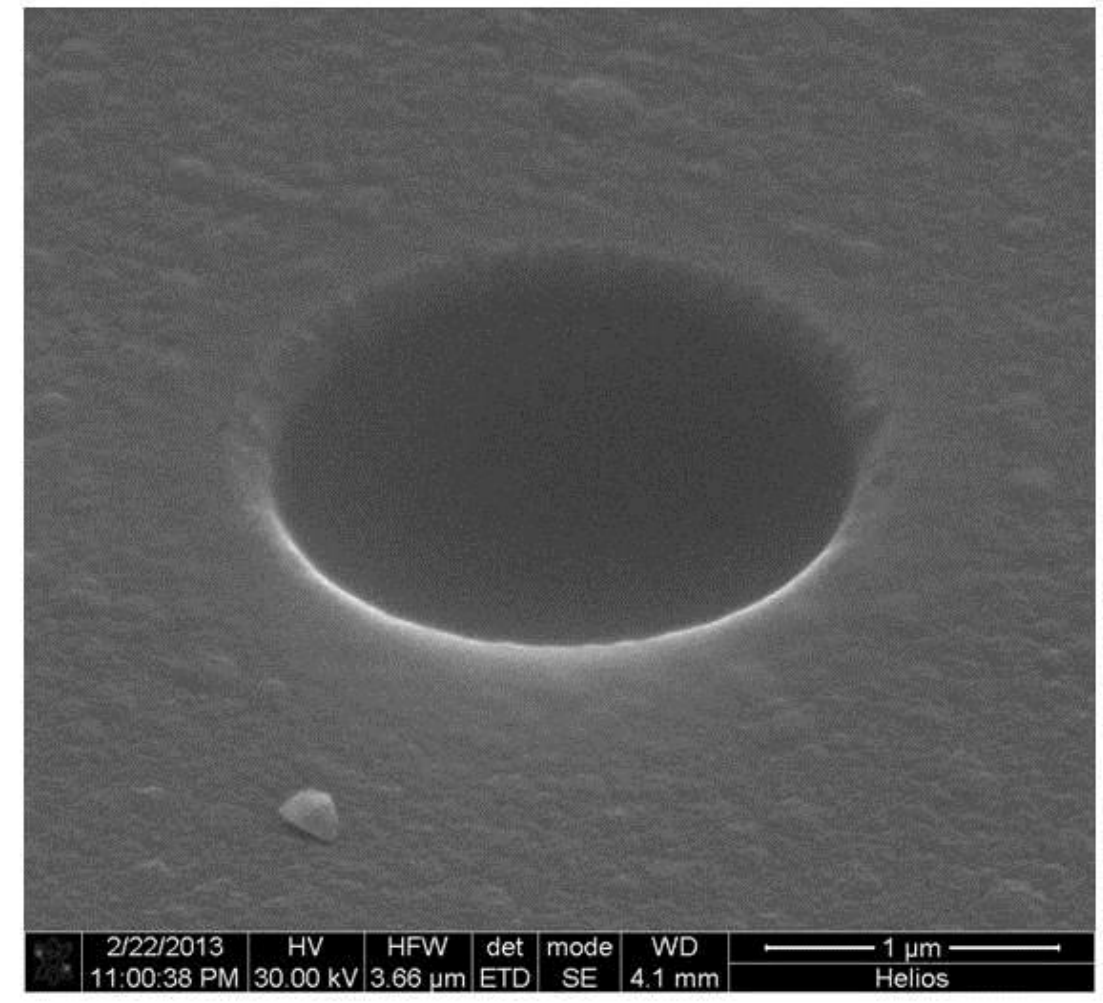

Fig. 7

(a)

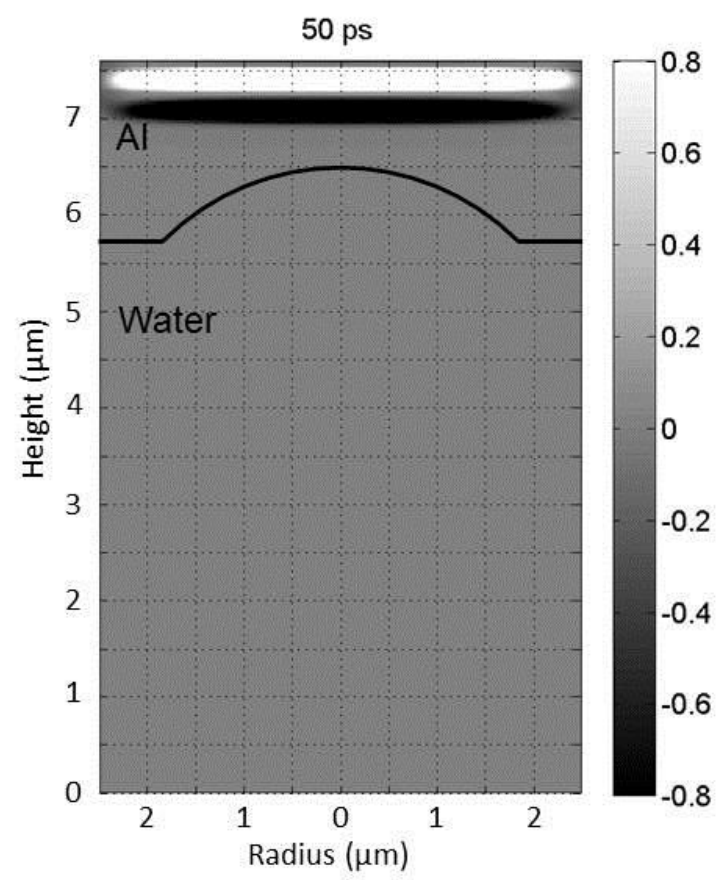

Fig. 8a 
(b)

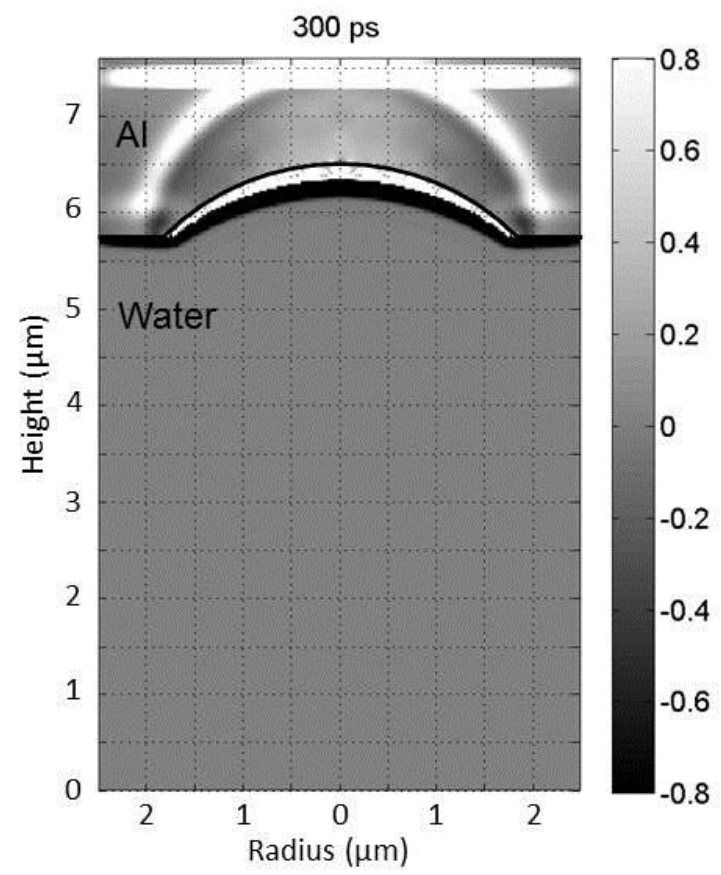

Fig. 8b

(c)

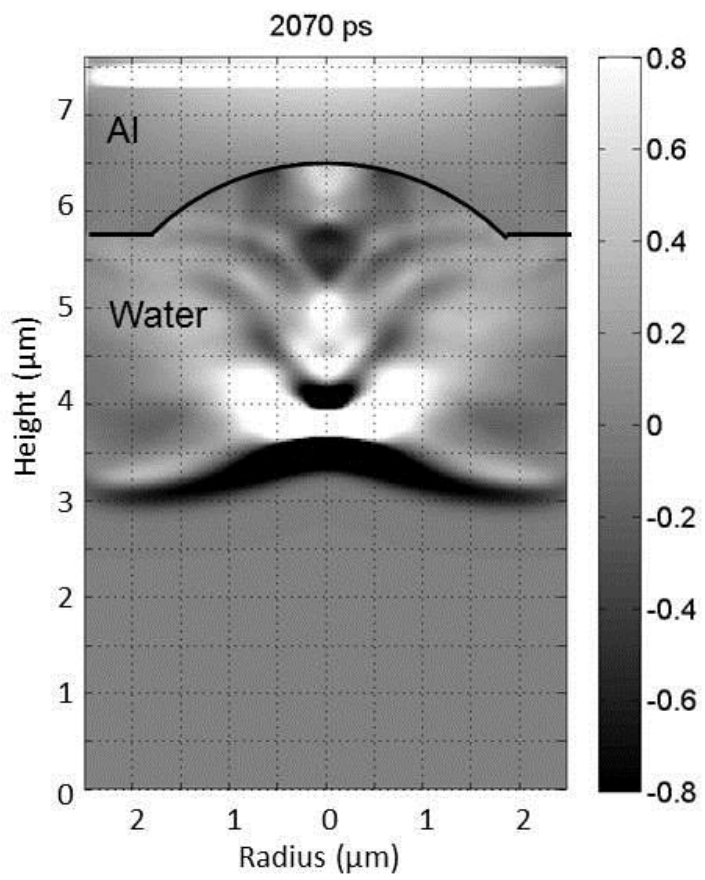

Fig. $8 \mathrm{c}$ 
(d)

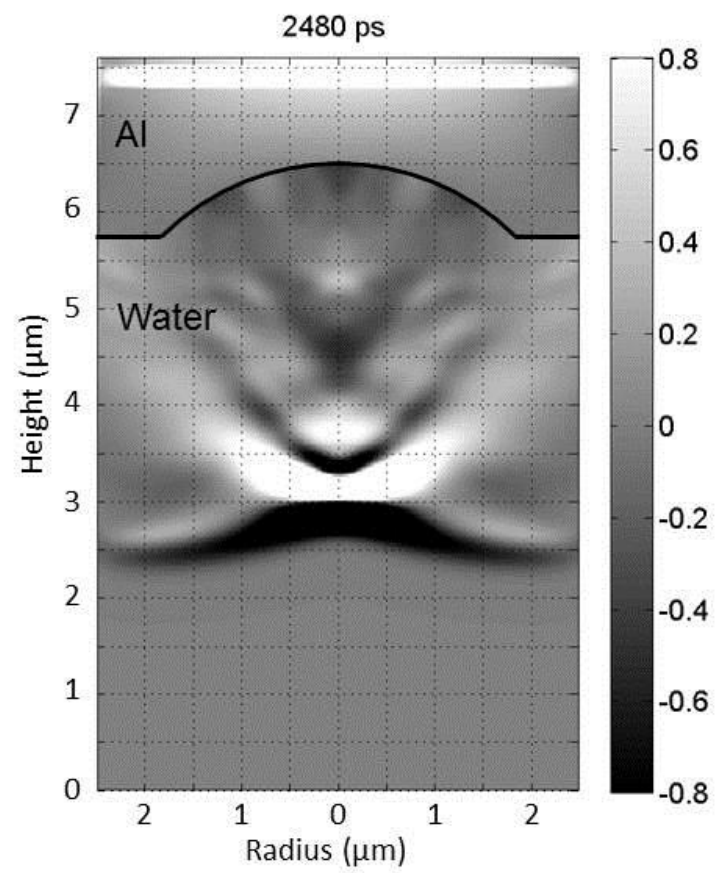

Fig. 8d

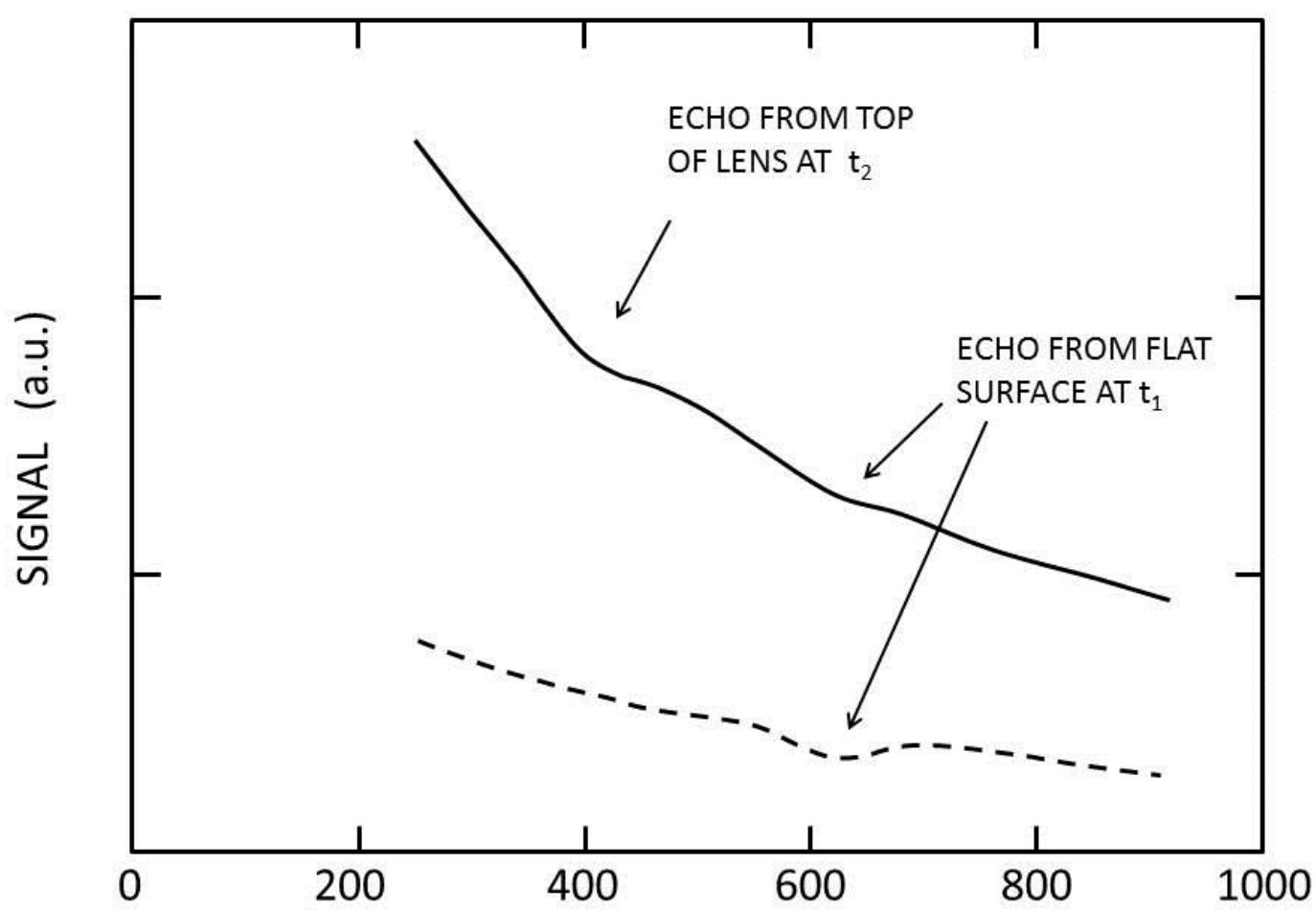

Fig. 9

PROBE DELAY TIME (ps) 


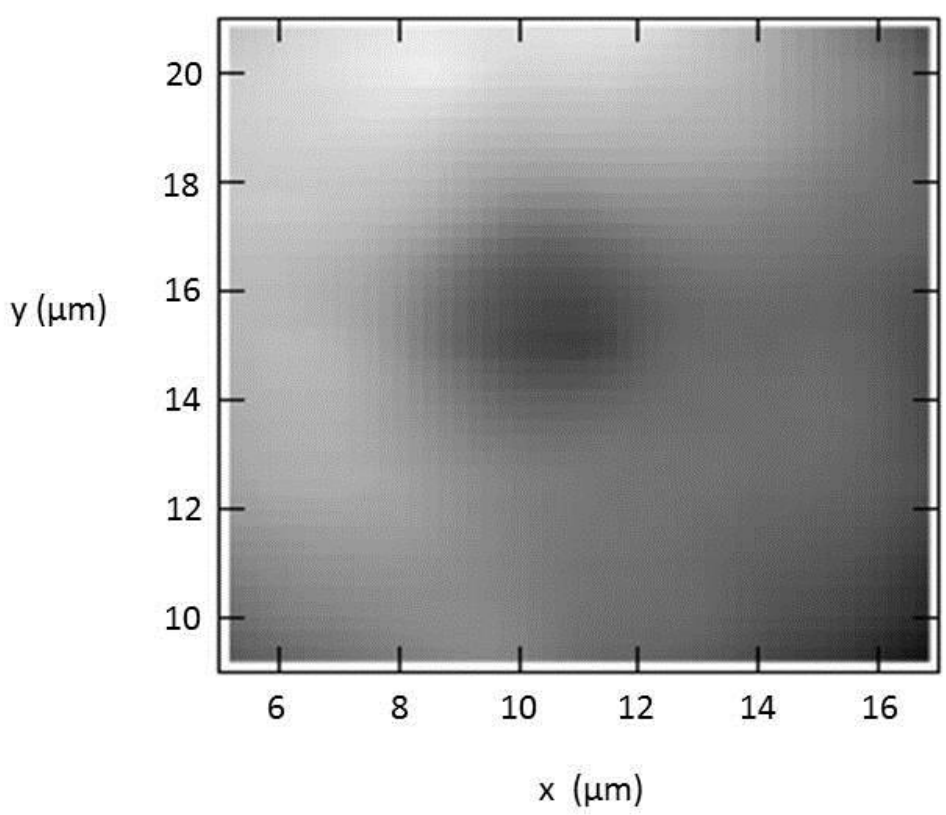

Fig. 10.

Fig. 11
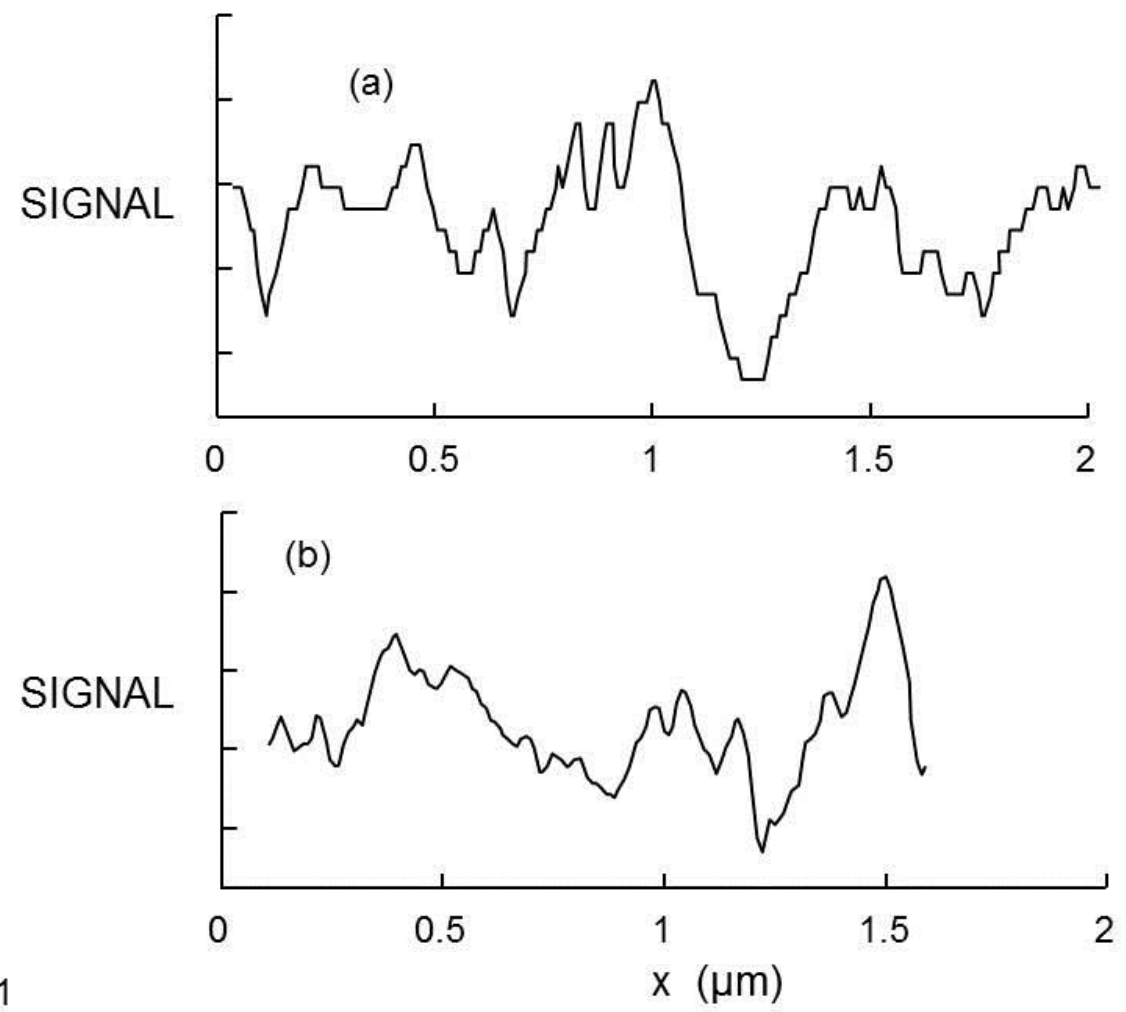


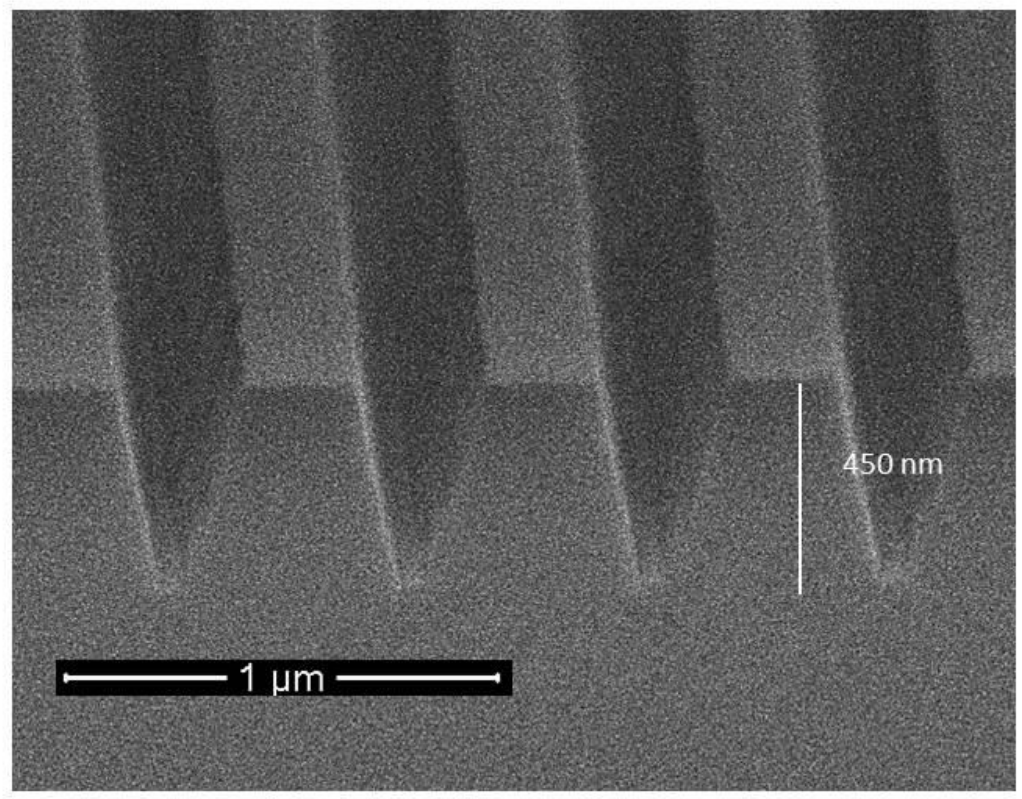

Fig. 12. 